CIRJE-F-539

\title{
Efficient Static Replication of European Options under Exponential Lévy Models
}

\author{
Akihiko Takahashi \\ University of Tokyo \\ Akira Yamazaki \\ Mizuho-DL Financial Technology Co., Ltd.
}

January 2008

CIRJE Discussion Papers can be downloaded without charge from:

http://www.e.u-tokyo.ac.jp/cirje/research/03research02dp.html

Discussion Papers are a series of manuscripts in their draft form. They are not intended for circulation or distribution except as indicated by the author. For that reason Discussion Papers may not be reproduced or distributed without the written consent of the author. 


\title{
Efficient Static Replication of European Options under Exponential Lévy Models*
}

\author{
Akihiko Takahashi $^{\dagger} \quad$ Akira Yamazaki ${ }^{\ddagger}$ \\ First Version: August 2007, This Version:January 2008
}

\begin{abstract}
This paper proposes a new scheme for the static replication of European options and their portfolios. First, we derive a general approximation formula for efficient static replication as an extension of Carr and Chou [1997, 2002] and Carr and Wu [2002]. Second, we present a concrete procedure for implementing our scheme by applying it to plain vanilla options under exponential Lévy models. Finally, numerical examples in a model developed by Carr, Geman, Madan and Yor[2002] are used to demonstrate that our replication scheme is more efficient and more effective in practice than a standard static replication method.
\end{abstract}

Keywords: Static Replication, Exponential Lévy Model, Fourier Transform Method, Plain Vanilla Option, CGMY Model

\footnotetext{
* forthcoming The Journal of Futures Markets

†The University of Tokyo

${ }_{\ddagger}$ Mizuho-DL Financial Technology Co., Ltd.
} 


\section{Introduction}

This paper develops a new efficient scheme for the static replication of European derivatives. Suppose the value of a target European derivative is twice differentiable in the underlying asset price; in other words, the gamma of the target derivative exists. By applying a technique similar to Carr and Chou [1997] and Carr and Madan [1998], we first show that the value of the derivative can be decomposed into a value-weighted bond, a delta-weighted forward contract and a gamma-weighted portfolio of options, all of whose maturities are shorter than the maturity of the target derivative. Based on this decomposition, a static replication can be obtained. However, theoretically an infinite number of options are needed for the replication. To overcome this problem, we introduce the Gauss-Legendre quadrature rule in order to approximate the replication based on a finite number of options. Consequently, compared with a standard static replication approach, our approach of gamma-weighted portfolio of options is more efficient; that is, a more precise hedge is derived from a smaller number of options.

To demonstrate this advantage, this paper presents a concrete procedure for implementing our scheme by applying it to a standard plain vanilla option under exponential Lévy models. Specifically, we derive semi-analytic formulas for the price, and the delta and gamma of the target option based on modifications of the fast Fourier transform method developed by Carr and Madan [1999]. In this way, we are able to achieve a very efficient computation for constructing static replication portfolios. It should also be noted that this scheme can be applied to other European derivatives such as cash digital, asset digital and power options.

Finally, when the underlying asset price dynamics is represented by a Carr, Geman, Madan and Yor [2002] (hereafter, CGMY) type exponential Lévy model that can describe the price processes in the real world very well, numerical examples show that our scheme significantly outperforms a standard static replication model. This result demonstrates that a more accurate replication can be derived from fewer options.

For over a decade, static hedging techniques have been developed and investigated extensively for barrier type options. Bowie and Carr [1994] and Carr, Ellis and Gupta [1998] consider a static hedge method for barrier-type and lookback options by using put call symmetry (Carr [1994]). Derman, Ergener and Kani [1995] proposes the calendar-spreads method. Carr and Picron [1999] presents a method for static hedging of timing risk which is applied to pricing barrier options.

Carr and Chou [1997, 2002] shows the representation of any twice differentiable payoff function that corresponds to lemma 1 in our paper. Their paper then develops the so called strike-spreads method for static hedging of barrier, ratchet and lookback options under the Black-Scholes model. Andersen, Andreasen and Eliezer [2002] theoretically investigates static replication of barrier options.

Fink [2003] generalizes the method of Derman, Ergener and Kani [1995] for barrier options in an environment of stochastic volatility. More recently, 
Nalholm and Poulsen [2006b] proposes a new technique for static hedging of barrier options under general asset dynamics, such as a jump-diffusion process with correlated stochastic volatility. Furthermore, Nalholm and Poulsen [2006a] examines the sensitivity of dynamic and static hedging methods for barrier options to model risk.

On the other hand, Carr and $\mathrm{Wu}$ [2002] concentrates on an efficient replication of a plain vanilla option though their approach implies the possibility of further extensions and applications. It also applies the Gauss-Hermite quadrature rule to approximate static hedging of the option by plain vanilla options with shorter terms under the Black-Scholes and Merton [1976] jump-diffusion models. Moreover, their paper undertakes extensive simulation exercises to investigate the robustness of the method. In a certain sense, our scheme relies on and extends the methodologies developed by Carr and Wu [2002], Carr and Chou [1997, 2002] and Carr and Madan [1998, 1999].

The remainder of the paper is organized as follows. The next section presents the details of our proposed method for static replication. Section 3 provides the formulas for the price, and the delta and gamma of a target option for replication under exponential Lévy models. In Section 4, numerical examples are applied to a CGMY model. The conclusions are presented in Section 5.

\section{Efficient Method for Static Replication}

This section presents a general efficient method for static replication of European options. Specifically, under a single factor Markovian setting, we develop a methodology to replicate European options and their portfolios based on a static portfolio of shorter term plain vanilla options. Static portfolio implies that the weights in the portfolio remain unchanged when the price of underlying assets moves and options in the portfolio approach maturity.

Under the assumptions of a frictionless and no arbitrage market, let $S_{t}$ denote the spot price of a stock, an underlying asset at time $t \in\left[0, T^{*}\right]$ where $T^{*}$ is some arbitrarily determined time horizon. For sake of simplicity, the interest rate $r$ and the dividend yield $d$ are assumed to be constants. The no-arbitrage condition ensures the existence of a risk-neutral probability measure $\mathbb{Q}$ defined on a probability space $(\Omega, \mathcal{F}, \mathbb{Q})$ such that the instantaneous expected rate of return on every asset is equal to the instantaneous interest rate $r$. Furthermore, the risk-neutral process of the underlying asset price is assumed to be timeinhomogeneous Markovian. Note that all exponential Lévy models belong to this class, where an exponential Lévy model implies that stock price dynamics are driven by Lévy processes. Moreover, the analysis in this paper concentrates on static replication of path-independent options where the final payoff of the option is solely determined by the stock price at maturity. Typical examples in this class include plain vanilla, cash digital, asset digital and power options.

The following formula implies that a static portfolio of plain vanilla options allows us to replicate any European derivatives under a certain condition. 
Lemma 1 Suppose that the payoff function $f\left(S_{T}\right)$ of a European derivative with maturity $T$ is twice differentiable. Then, for any $\kappa>0$, it satisfies

$$
\begin{aligned}
f\left(S_{T}\right) & =f(\kappa)+f^{\prime}(\kappa)\left(S_{T}-\kappa\right) \\
& +\int_{0}^{\kappa} f^{\prime \prime}(K)\left(K-S_{T}\right)^{+} d K+\int_{\kappa}^{+\infty} f^{\prime \prime}(K)\left(S_{T}-K\right)^{+} d K .
\end{aligned}
$$

Moreover, for all $t \in[0, T]$ the present value $V_{t}\left(S_{t}\right)$ of the derivative satisfies

$$
\begin{aligned}
V_{t}\left(S_{t}\right) & =e^{-r(T-t)} f(\kappa)+e^{-r(T-t)} f^{\prime}(\kappa)\left\{F_{t}(T)-\kappa\right\} \\
& +\int_{0}^{\kappa} f^{\prime \prime}(K) P_{t}(T, K) d K+\int_{\kappa}^{+\infty} f^{\prime \prime}(K) C_{t}(T, K) d K,
\end{aligned}
$$

where $F_{t}(T)$ denotes the time-t price of the forward contract with maturity $T$, and $P_{t}(T, K):=P_{t}\left(S_{t} ; T, K\right)$ and $C_{t}(T, K):=C_{t}\left(S_{t} ; T, K\right)$ represent the time$t$ prices of plain vanilla put and call options with spot price $S_{t}$, strike $K$ and maturity $T$ respectively.

Proof: See Carr and Chou [1997] or Appendix 1 in Carr and Madan [1998] for instance.

The following proposition indicates that an European derivative can be replicated by using plain vanilla options whose maturities are shorter than the target European derivative so long as the delta and gamma for all possible values of the underlying spot price exist. Because the price function of a derivative can be regarded as a payoff function, the proof of the proposition is obvious from Lemma 1.

Proposition 1 Let $\tau \in[0, T]$. Suppose that the time- $\tau$ price function $V_{\tau}(S)$ of a European derivative with maturity $T$ is twice differentiable for all $S \geq 0$, that $i s$, both the delta and gamma of the derivative exist at time $\tau$. For any $\kappa>0$, it satisfies

$$
\begin{aligned}
V_{\tau}\left(S_{\tau}\right) & =V_{\tau}(\kappa)+\left.\frac{\partial V_{\tau}}{\partial S}\right|_{S=\kappa}\left(S_{\tau}-\kappa\right) \\
& +\left.\int_{0}^{\kappa} \frac{\partial^{2} V_{\tau}}{\partial S^{2}}\right|_{S=K}\left(K-S_{\tau}\right)^{+} d K+\left.\int_{\kappa}^{+\infty} \frac{\partial^{2} V_{\tau}}{\partial S^{2}}\right|_{S=K}\left(S_{\tau}-K\right)^{+} d K .
\end{aligned}
$$

Moreover, for all $t \in[0, \tau]$ the present value $V_{t}\left(S_{t}\right)$ of the derivative satisfies 


$$
\begin{aligned}
V_{t}\left(S_{t}\right) & =e^{-r(\tau-t)} V_{\tau}(\kappa)+\left.e^{-r(\tau-t)} \frac{\partial V_{\tau}}{\partial S}\right|_{S=\kappa}\left\{F_{t}(\tau)-\kappa\right\} \\
& +\left.\int_{0}^{\kappa} \frac{\partial^{2} V_{\tau}}{\partial S^{2}}\right|_{S=K} P_{t}(\tau, K) d K+\left.\int_{\kappa}^{+\infty} \frac{\partial^{2} V_{\tau}}{\partial S^{2}}\right|_{S=K} C_{t}(\tau, K) d K
\end{aligned}
$$

According to Proposition 1, once the replication portfolio is created, re-balancing is unnecessary until the maturity date of the options in the portfolio. This property is called static. Note that although nearly none of the payoff functions of the derivatives are twice differentiable, their price functions are mostly twice differentiable. Hence, Proposition 1 is more useful for applications. The practical implication of this proposition is that the risk embedded in a target European derivative can be hedged using a static portfolio of liquid plain vanilla options with a maturity that is shorter than the maturity of the target derivative.

Next, we present an efficient method for static replication. Proposition 1 shows that any derivative whose price function is twice differentiable can be completely replicated by using an infinite number of plain vanilla options. However, since an infinite number of options can not be used in practice, approximation of a static portfolio using a finite number of the options is necessary. Specifically, we apply the Gauss-Legendre quadrature rule for the approximation. The rule is a numerical computational method for an integral $\int_{-1}^{1} g(x) d x$, where $g(x) \in C^{2 n}(n \in \mathbf{N})$ on $[-1,1]$. Here, $C^{2 n}$ denotes the set of $2 n$-times continuously differentiable functions. For a given target function $g(x)$, the GaussLegendre quadrature rule provides the following formula.

$$
\int_{-1}^{1} g(x) d x=\sum_{j=1}^{n} \omega_{j} g\left(x_{j}\right)+\frac{2^{2 n+1}(n !)^{4}}{(2 n+1)[(2 n) !]^{3}} g^{(2 n)}(\xi),
$$

for some $\xi \in[-1,1]$, where $x_{j}, j=1,2, \cdots, n$, are roots of the $n$th order Legendre polynomial $L_{n}(x), \omega_{j}:=2 /\left(n L_{n-1}\left(x_{j}\right) L_{n}^{\prime}\left(x_{j}\right)\right)$ and $g^{(2 n)}$ denotes the $2 n$-th derivative of $g$. The second term on the right hand side of equation (5) is the approximation error on the $n$-th order Gauss-Legendre quadrature rule. Note that if $g(x)$ is smooth, the error term converges to zero when $n \rightarrow \infty$. For details of the Gaussian quadrature rule, see pp. 225-230 of Sugihara and Murota [1994] for example. Application of the Gauss-Legendre quadrature rule to Proposition 1 provides the main result in this paper, which can be stated as the following theorem.

Theorem 1 Let $\tau \in[0, T]$, and suppose that $C_{t}(\tau, K) \in C^{2 m}$ and $P_{t}(\tau, K) \in$ $C^{2 n}$ with respect to $K$ respectively. Let $V_{\tau}(S) \in C^{q}$ be the time- $\tau$ price function of an European derivative with maturity $T$ where $q:=2 \max \{m, n\}$. Assume that there exist $S_{\min } \in[0, \kappa)$ and $S_{\max } \in(\kappa, \infty)$ such that for all $t \in[0, \tau]$ 


$$
\begin{array}{ll}
\left.\frac{\partial^{2} V_{\tau}}{\partial S^{2}}\right|_{S=y} P_{t}(\tau, y)=0 & \text { if } y \in\left[0, S_{\min }\right], \\
\left.\frac{\partial^{2} V_{\tau}}{\partial S^{2}}\right|_{S=z} C_{t}(\tau, z)=0 & \text { if } z \in\left[S_{\max },+\infty\right) .
\end{array}
$$

Moreover, define a static portfolio $\Lambda_{t}(n, m)$ as follows:

$$
\begin{aligned}
\Lambda_{t}(n, m):= & e^{-r(\tau-t)} V_{\tau}(\kappa)+\left.e^{-r(\tau-t)} \frac{\partial V_{\tau}}{\partial S}\right|_{S=\kappa}\left\{F_{t}(\tau)-\kappa\right\} \\
& +\sum_{j=1}^{n} A_{j}^{P} P_{t}\left(\tau, K_{j}^{P}\right)+\sum_{l=1}^{m} A_{l}^{C} C_{t}\left(\tau, K_{l}^{C}\right),
\end{aligned}
$$

where

$$
\begin{aligned}
& K_{j}^{P}:=\frac{\kappa-S_{\min }}{2} x_{j}^{n}+\frac{\kappa+S_{\min }}{2}, \quad A_{j}^{P}:=\left.\omega_{j}^{n}\left(\frac{\kappa-S_{\min }}{2}\right) \frac{\partial^{2} V_{\tau}}{\partial S^{2}}\right|_{S=K_{j}^{P}}, \\
& K_{l}^{C}:=\frac{S_{\max }-\kappa}{2} x_{l}^{m}+\frac{S_{\max }+\kappa}{2}, \quad A_{l}^{C}:=\left.\omega_{l}^{m}\left(\frac{S_{\max }-\kappa}{2}\right) \frac{\partial^{2} V_{\tau}}{\partial S^{2}}\right|_{S=K_{l}^{C}}, \\
& \omega_{j}^{n}:=\frac{2}{n L_{n-1}\left(x_{j}^{n}\right) L_{n}^{\prime}\left(x_{j}^{n}\right)}, \quad \omega_{l}^{m}:=\frac{2}{m L_{m-1}\left(x_{l}^{m}\right) L_{m}^{\prime}\left(x_{l}^{m}\right)} .
\end{aligned}
$$

Here, $x_{j}^{n}, j=1, \cdots, n\left(x_{l}^{m}, l=1, \cdots, m\right)$ denote the roots of the $n$-th $(m-$ th $)$ order Legendre polynomial.

Then, $\Lambda_{t}(n, m)$ approximates $V_{t}\left(S_{t}\right)$ for all $t \in[0, \tau]$ :

$$
V_{t}\left(S_{t}\right)=\Lambda_{t}(n, m)+p_{n}\left(\xi_{1}\right)+p_{m}\left(\xi_{2}\right) \text {, for some } \xi_{1}, \xi_{2} \in[-1,1],
$$

where $p_{n}\left(\xi_{1}\right)\left(p_{m}\left(\xi_{2}\right)\right)$ is the error term of the $n$-th (m-th) order quadrature rule.

In particular, if $C_{t}(\tau, K), P_{t}(\tau, K)$ and $V_{\tau}(S)$ are smooth, $\Lambda_{t}(n, m)$ converges to $V_{t}\left(S_{t}\right)$ for all $t \in[0, \tau]$, when $n \rightarrow+\infty$ and $m \rightarrow+\infty$.

Proof: Let us define the following integral.

$$
I:=\left.\int_{0}^{\kappa} \frac{\partial^{2} V_{\tau}}{\partial S^{2}}\right|_{S=K} P_{t}(\tau, K) d K=\left.\int_{S_{\min }}^{\kappa} \frac{\partial^{2} V_{\tau}}{\partial S^{2}}\right|_{S=K} P_{t}(\tau, K) d K
$$

Changing the integral parameter $K$ into $\frac{\kappa-S_{\min }}{2} x+\frac{\kappa+S_{\min }}{2}$, we re-write the integral (10) as

$$
\begin{aligned}
I= & \frac{\kappa-S_{\min }}{2} \\
& \times\left.\int_{-1}^{1} \frac{\partial^{2} V_{\tau}}{\partial S^{2}}\right|_{S=\frac{\kappa-S_{\min }}{2} x+\frac{\kappa+S_{\min }}{2}} P_{t}\left(\tau, \frac{\kappa-S_{\min }}{2} x+\frac{\kappa+S_{\min }}{2}\right) d x .
\end{aligned}
$$


Then, the Gauss-Legendre quadrature rule can be applied to the integral (11). That is,

$$
I=\sum_{j=1}^{n} A_{j}^{P} P_{t}\left(\tau, K_{j}^{P}\right)+p_{n}\left(\xi_{1}\right), \quad \text { for some } \xi_{1} \in[-1,1],
$$

where $p_{n}(\xi)$ denotes the error term of the $n$-th order quadrature rule.

A similar argument holds for:

$$
J:=\left.\int_{\kappa}^{+\infty} \frac{\partial^{2} V_{\tau}}{\partial S^{2}}\right|_{S=K} C_{t}(\tau, K) d K=\left.\int_{\kappa}^{S_{\max }} \frac{\partial^{2} V_{\tau}}{\partial S^{2}}\right|_{S=K} C_{t}(\tau, K) d K .
$$

Further, if $C_{t}(\tau, K), P_{t}(\tau, K)$ and $V_{\tau}(S)$ are smooth, it clearly holds that when $n \rightarrow+\infty, m \rightarrow+\infty$,

$$
\left.\sum_{j=1}^{n} A_{j}^{P} P_{t}\left(\tau, K_{j}^{P}\right) \longrightarrow \int_{0}^{\kappa} \frac{\partial^{2} V_{\tau}}{\partial S^{2}}\right|_{S=K} P_{t}(\tau, K) d K,
$$

and

$$
\left.\sum_{l=1}^{m} A_{l}^{C} C_{t}\left(\tau, K_{l}^{C}\right) \longrightarrow \int_{\kappa}^{+\infty} \frac{\partial^{2} V_{\tau}}{\partial S^{2}}\right|_{S=K} C_{t}(\tau, K) d K
$$

Remark 1 Although assumption (6) in the theorem may not hold rigorously in applications, a static portfolio $\Lambda_{t}(n, m)$ is very effective because the gamma of most European derivatives, such as plain vanilla, cash digital and asset digital options, approaches zero very quickly as the moneyness goes to in-the-money and out-of-the-money.

\section{Option Prices and Greeks under Exponential Lévy Models}

This section derives the formulas for the price and the Greeks of a European plain vanilla option under exponential Lévy models. This is done because, in practical situations, efficient and accurate computation of the price and Greeks is crucial for static replication.

Suppose the stock price process is specified as $S_{t}=S_{0} e^{(r-d) t+X_{t}}, t \in\left[0, T^{*}\right]$ under a risk-neutral measure $\mathbb{Q}$, where $\left(X_{t}\right)_{t \geq 0}$ is a one-dimensional stochastic process with $X_{0}=0$ and is an exponential martingale on the probability space $(\Omega, \mathcal{F}, P)$ endowed with a standard complete filtration $\mathbb{F}:=\left(\mathcal{F}_{t}\right)_{t \geq 0}$. In particular, $\left(X_{t}\right)_{t>0}$ is assumed to be a Lévy process with respect to the filtration $\mathbb{F}$. By the Lévy Khintchine formula (see Sato [1991] for example), the characteristic function of $X_{t}$ takes the form 


$$
\Phi_{X_{t}}(\theta):=\mathbb{E}\left[e^{i \theta X_{t}}\right]=e^{-t \psi_{X}(\theta)}, \quad t \geq 0,
$$

where the characteristic exponent $\psi_{X}(\theta), \theta \in \mathbf{R}$, is given by

$$
\psi_{X}(\theta)=-i \gamma \theta+\frac{1}{2} \sigma^{2} \theta^{2}+\int_{-\infty}^{+\infty}\left(1-e^{i \theta x}+i \theta x \mathbf{1}_{|x| \leq 1}\right) \Pi(d x),
$$

where $\sigma \geq 0$ and $\gamma \in \mathbf{R}$ are constants, and $\Pi$ is a measure on $\mathbf{R} \backslash\{0\}$ satisfying

$$
\int_{-\infty}^{+\infty}\left(1 \wedge x^{2}\right) \Pi(d x)<+\infty .
$$

In many exponential Lévy models, the characteristic function $\Phi_{X_{t}}(\theta)$ can be obtained analytically. Indeed, in the case of the Black-Scholes model (i.e. $S_{t}=$ $S_{0} e^{(r-d) t-\frac{1}{2} \sigma^{2}+\sigma W_{t}}$, where $W_{t}$ is a one-dimensional standard Brownian motion and $\sigma$ is a volatility) the characteristic function $\Phi_{B S_{t}}^{\sigma}(\theta)$ of $B S_{t}:=-\frac{1}{2} \sigma^{2}+\sigma W_{t}$ is given by $\Phi_{B S_{t}}^{\sigma}(\theta)=\exp \left\{-\frac{\sigma^{2} t}{2}\left(\theta^{2}+i \theta\right)\right\}$. Other well-known examples are the characteristic functions of Merton's jump-diffusion process (Merton [1976]), Kou's jump-diffusion process (Kou [2002]), the Variance Gamma process (Mardan, Carr and Chang [1998]), the normal inverse Gaussian process (BarndorffNielsen [1997]), the CGMY process (Carr, Geman, Madan and Yor [2002]), the generalized hyperbolic process (Eberlein, Keller and Prause [1998]), and the finite moment log-stable process (Carr and Wu [2003]).

Carr and Madan [1999] introduces a fast Fourier transform method for option pricing. This paper proposes to compute the time value of the option after subtracting an intrinsic value from the option price in order to avoid the oscillation of the integrand in the Fourier inversion. As a result, the option price can be obtained as the time value derived by the Fourier inversion plus the intrinsic value. On the other hand, to compute the delta and gamma of an option, we propose to subtract the Black-Scholes price with appropriate volatility from the option price instead of subtracting the intrinsic value. This choice is made because the intrinsic value might not be differentiable. See also p.363 of Cont and Tankov [2003]. (Note that the Black-Scholes prices of European options are twice differentiable.)

The following proposition shows the formulas for the price, the delta and the gamma of a plain vanilla call option.

Proposition 2 Let $C_{t}$ denote a plain vanilla call price with strike $K$ and maturity $T$ at time $t$. Then the call price is given by

$$
C_{t}=\frac{S e^{-\alpha k}}{2 \pi} \int_{-\infty}^{+\infty} e^{-i u k} \zeta_{T, t}(u) d u+C_{t}^{\sigma}
$$

where 


$$
\begin{aligned}
\zeta_{T, t}(u):= & \frac{\exp \{[(r-d)(i u+\alpha+1)-r](T-t)\}}{(i u+\alpha)(i u+\alpha+1)} \\
& \times\left(\Phi_{X_{T-t}}(u-i \alpha-i)-\Phi_{B S_{T-t}}^{\sigma}(u-i \alpha-i)\right),
\end{aligned}
$$

$\alpha>0, k:=\ln (K / S)$ and $C_{t}^{\sigma}$ denotes the Black-Scholes price of the plain vanilla call with some volatility $\sigma>0$. Moreover, the delta $\frac{\partial C_{t}}{\partial S}$ and the gamma $\frac{\partial^{2} C_{t}}{\partial S^{2}}$ are given by

$$
\begin{aligned}
\frac{\partial C_{t}}{\partial S} & =\frac{e^{-\alpha k}}{2 \pi} \int_{-\infty}^{+\infty}(i u+\alpha+1) e^{-i u k} \zeta_{T, t}(u) d u+\frac{\partial C_{t}^{\sigma}}{\partial S}, \\
\frac{\partial^{2} C_{t}}{\partial S^{2}} & =\frac{e^{-\alpha k}}{2 \pi S} \int_{-\infty}^{+\infty}(i u+\alpha)(i u+\alpha+1) e^{-i u k} \zeta_{T, t}(u) d u+\frac{\partial^{2} C_{t}^{\sigma}}{\partial S^{2}} .
\end{aligned}
$$

Proof: See Appendix.

\section{Numerical Examples}

This section examines the effectiveness of our replication scheme through numerical examples. First, let us specify the stock process $S_{t}=S_{0} e^{(r-d) t+X_{t}}$ as the CGMY model under a risk-neutral measure $\mathbb{Q}$, which is introduced by Carr, Geman, Madan and Yor [2002]. That is, $X_{t}:=\omega t+Z_{t}^{C G M Y}$ where $\omega:=-\psi_{Z^{C G M Y}}(-i)$. Here, $\omega$ is called a convexity correction, and $Z_{t}^{C G M Y}$ is a pure jump Lévy process whose Lévy measure $\Pi_{C G M Y}$ is defined by:

$$
\Pi_{C G M Y}(d x):= \begin{cases}C \frac{\exp \{-G|x|\}}{|x|^{1+Y}} d x & \text { for } x<0 \\ C \frac{\exp \{-M|x|\}}{|x|^{1+Y}} d x & \text { for } x>0,\end{cases}
$$

where $C>0, G \geq 0, M \geq 0$, and $Y<2$. The characteristic exponent of $Z_{t}^{C G M Y}$ is given by

$$
\psi_{Z^{C G M Y}}(\theta)=C \Gamma(-Y)\left[M^{Y}-(M-i \theta)^{Y}+G^{Y}-(G+i \theta)^{Y}\right],
$$

where $\Gamma(\cdot)$ is the gamma function. See Carr, Geman, Madan and Yor [2002] for details.

The input parameters of the CGMY model in the numerical examples are listed in Table 1, where the CGMY parameters are taken from Table 2 in Carr, Geman, Madan and Yor [2002]. 
Table 1: The input parameters of the CGMY model

\begin{tabular}{ccccccc}
\hline$S_{0}$ & $r$ & $d$ & $C$ & $G$ & $M$ & $Y$ \\
\hline 100 & 0.00 & 0.00 & 24.79 & 94.45 & 95.79 & 0.2495 \\
\hline
\end{tabular}

In order to examine effectiveness of our method, we compare it to a static replication approach that is referred to as the standard method in the subsequent analysis. In the standard method, the replication portfolio for a given target option consists of various plain vanilla options with different strike prices and the same maturity, which is shorter than the maturity of the target option. The replication portfolio is obtained so that the value of the portfolio is equal to that of the target option for each discretized grid of stock prices at the maturity of the portfolio. At each grid, the corresponding portfolio weights can be found by solving a system of linear equations. The general procedure of the standard method can be found in Nalholm and Poulsen[2006b], for instance. On the other hand, our replication scheme is hereafter referred to as the efficient method.

We compute the replication portfolios for a target plain vanilla call with strike $K=100$ and maturity $T=1$. Using 8,12 , or 16 plain vanilla options with maturity $\tau=0.5$, we replicate each value of the target option for all $S_{t} \in[50,150], 0 \leq t \leq \tau$. Figure 1 describes the present values of the target option with different underlying stock prices and times-to-expiry. Figures 24 plot the errors of the replication that are defined as the deviations of the portfolio's values from the target option's values. It is obvious that the efficient method provides more accurate approximations of the replication portfolio than the standard method in all cases. Table 2 shows the costs of replication that are equivalent to the portfolio values at the initial time, as well as the errors and error ratios against the corresponding option premium. In the efficient method, considerable accuracy in prices can be obtained by using only 8 options. Consequently, these numerical results show that the efficient method is effective and efficient in practice.

Remark 2 A similar technique was applied to the static replication of European derivatives such as cash digital, asset digital and power options. We obtained particularly good results for these options in numerical experiments using the same CGMY model. The results will be made available upon request.

\section{Conclusion}

This paper presents a new scheme for the static replication of European derivatives under a general class of exponential Lévy models. The scheme can be applied to European derivatives including digital-type options for which dynamic hedging is sometimes difficult to implement and is therefore not very effective in practice. Our efficient method developed in a general class of the underlying price process appears to be useful and widely applicable in trading 
and hedging of derivatives. Moreover, numerical examples in a CGMY model confirm the validity of our scheme through comparison with a standard static replication method. Finally, our next research topic will be to establish an effective and efficient scheme for the static replication of multi-factor derivatives, such as stochastic volatility models.

Table 2: The cost of replication for the target call option

\begin{tabular}{l|r|r|r}
\hline Exact Value & \multicolumn{3}{|c}{4.86176} \\
\hline Number of Options & 8 & 12 & 16 \\
\hline Efficient Method & 4.86424 & 4.86167 & 4.86175 \\
Error & -0.00248 & 0.00010 & 0.00001 \\
Error Ratio & $-0.0510 \%$ & $0.0020 \%$ & $0.0003 \%$ \\
\hline Standard Method & 5.21137 & 4.95555 & 4.89037 \\
Error & -0.34961 & -0.09379 & -0.02861 \\
Error Ratio & $-7.1910 \%$ & $-1.9291 \%$ & $-0.5884 \%$ \\
\hline
\end{tabular}


Figure 1: The present value of the target call option

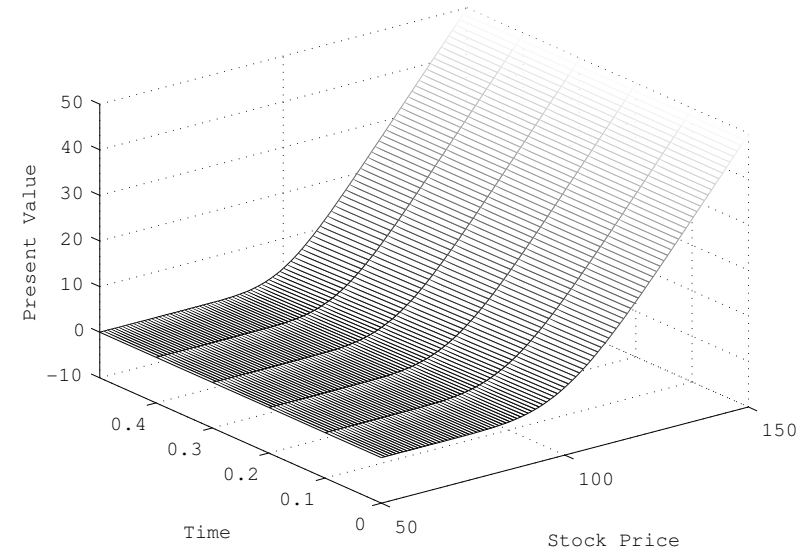

Figure 2: The replication error with 8 options
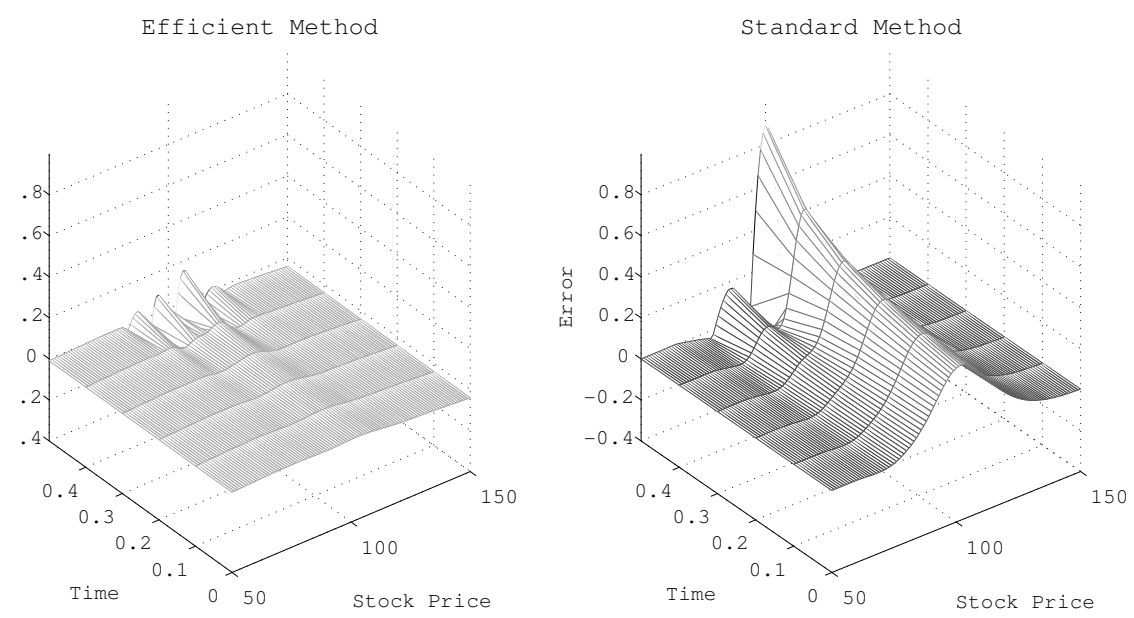
Figure 3: The replication error with 12 options
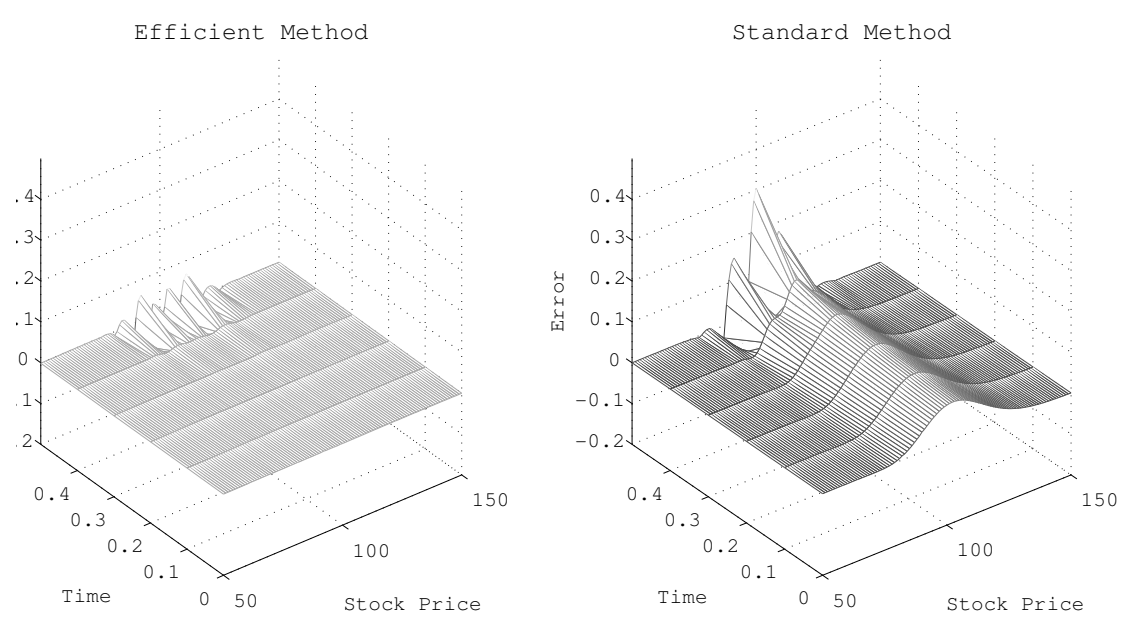

Figure 4: The replication error with 16 options
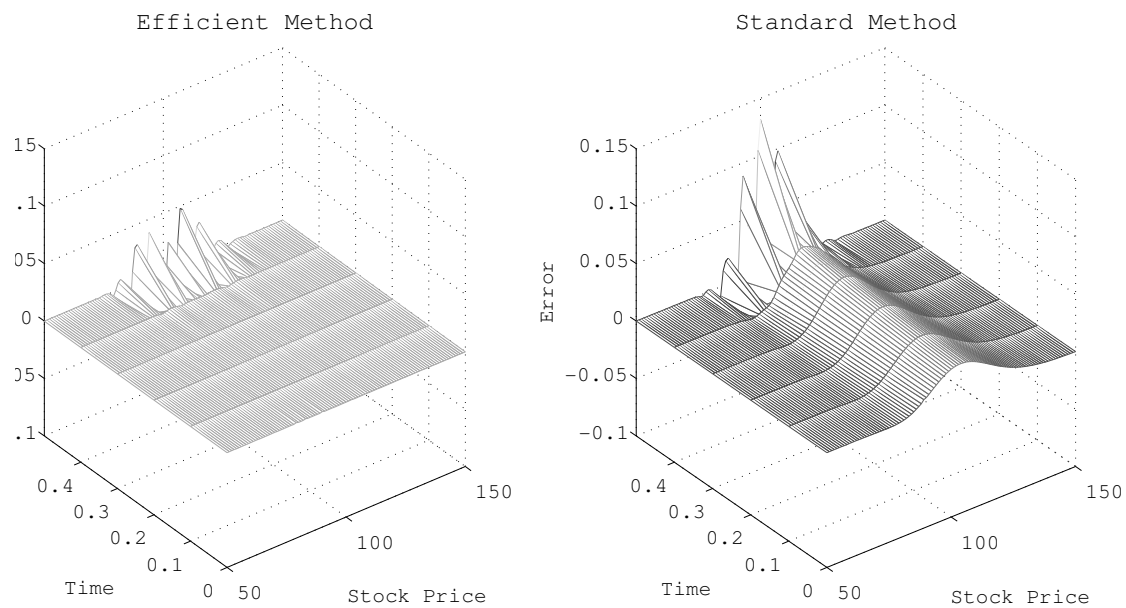


\section{References}

[1] Andersen, L., Andreasen, J., and Eliezer, D. Static replication of barrier options: Some general results. Journal of Computational Finance, 5(4):1$25,2002$.

[2] Barndorff-Nielsen, O. E. Process of normal inverse Gaussian type. Finance ans Stochastics, 2:41-68, 1997.

[3] Bowie, J., and Carr, P. Static simplicity. Risk, 7(8):45-49, 1994.

[4] Boyarchenko, S., and Levendorskii, S. Non-Gaussian Merton-Black-Scholes Theory. World Scientific, 2002.

[5] Carr, P. European put call symmetry. Working Paper, Cornell University, 1994.

[6] Carr, P., and Chou, A. Breaking barriers. Risk, 10(9):139-145, 1997.

[7] Carr, P., and Chou, A. Hedging complex barrier options. Working Paper, 2002.

[8] Carr, P., and Madan, D. Towards a theory of volatility trading. In R. Jarrow, editor, Volatility, pages 417-427. Risk Publications, 1998.

[9] Carr, P., and Madan, D. Option valuation using the fast Fourier transform. Journal of Computational Finance, 2(4):61-73, 1999.

[10] Carr, P., and Picron, J. Static hedging of timing risk. Journal of Derivatives, pages 57-70, Spring 1999.

[11] Carr, P., and Wu, L. Static hedging of standard options. Working Paper, 2002.

[12] Carr, P., and Wu, L. Finite moment log stable process and option pricing. Journal of Finance, 58:753-777, 2003.

[13] Carr, P., Ellis, K., and Gupta, V. Static hedging of exotic options. Journal of Finance, 53:1165-1190, 1998.

[14] Carr, P., Geman, H., Madan, D., and Yor, M. The fine structure of asset returns: An empirical investigation. Journal of Business, 75:305-332, 2002.

[15] Cont, R., and Tankov. P. Financial Modelling with Jump Processes. Chapman \& HALL/CRC, 2003.

[16] Derman, E., Ergener, D., and Kani, I. Static options replication. Journal of Derivatives, pages 78-95, Summer 1995.

[17] Eberlein, E., Keller, U., and Prause, K. New insights into smile, mispricing, and value at risk: The hyperbolic model. Management Science, 71:371-406, 1998. 
[18] Fink, J. An examination of the effectiveness of static hedging in the presence of stochastic volatility. Journal of Futures Markets, 23(9):859-890, 2003.

[19] Kou, S. A jump-diffusion model for option pricing. Management Science, 48:1086-1101, 2002.

[20] Madan, D., Carr, P., and Chang, E. C. Variance gamma process and option pricing. European Finance Review, 2:79-105, 1998.

[21] Merton, R. Option pricing when underlying stock returns are discontinuous. Journal of Financial Economics, 3:125-144, 1976.

[22] Nalholm, M., and Poulsen, R. Static hedging and model risk for barrier options. Journal of Futures Markets, 26(5):449-463, 2006a.

[23] Nalholm, M., and Poulsen, R. Static hedging of barrier options under general asset dynamics: Unification and application. Journal of Derivatives, pages 46-60, Summer 2006b.

[24] Sato, K. Lévy Processes and Infinitely Divisible Distributions. Cambridge University Press, 1991.

[25] Schoutens, W. Exotic options under Lévy models: An overview. Working Paper, 2004.

[26] Sugihara, M., and Murota, K. Mathematics of Numerical Computation (Suchi Keisan Hou no Suri (in Japanese)). Iwanami, 1994. 


\section{Appendix: The Proof of Proposition 2}

Note that

$$
\begin{aligned}
C_{t} & =\mathbb{E}\left[e^{-r(T-t)}\left(S_{T}-K\right)^{+} \mid \mathcal{F}_{t}\right] \\
& =S_{t} e^{-r \bar{T}} \int_{-\infty}^{\infty}\left(e^{(r-d) \bar{T}+x}-e^{k}\right) \mathbf{1}_{\{(r-d) \bar{T}+x>k\}} \rho_{X_{\bar{T}}}(x) d x,
\end{aligned}
$$

where $\bar{T}:=T-t$ and $\rho_{X_{\bar{T}}}(\cdot)$ is the density function of $X_{\bar{T}}$. We define the function $\bar{\zeta}_{T, t}(k)$ as

$$
\begin{aligned}
\bar{\zeta}_{T, t}(k):= & \frac{e^{\alpha k}}{S_{t}}\left(C_{t}-C_{t}^{\sigma}\right) \\
= & e^{-r \bar{T}+\alpha k} \int_{-\infty}^{\infty}\left(\rho_{X_{\bar{T}}}(x)-\rho_{B S_{\bar{T}}}(x)\right) \\
& \times\left(e^{(r-d) \bar{T}+x}-e^{k}\right) \mathbf{1}_{\{(r-d) \bar{T}+x>k\}} d x .
\end{aligned}
$$

Let $\zeta_{T, t}(u)$ denote the Fourier transform of $\bar{\zeta}_{T, t}(k)$. Then, $\zeta_{T, t}(u)$ can be calculate as follows:

$$
\begin{aligned}
\zeta_{T, t}(u)= & \int_{-\infty}^{\infty} e^{i u k} \bar{\zeta}_{T, t}(k) d k \\
= & e^{-r \bar{T}} \int_{-\infty}^{\infty}\left(\rho_{X_{\bar{T}}}(x)-\rho_{B S_{\bar{T}}}(x)\right) \\
& \times \int_{-\infty}^{(r-d) \bar{T}+x} e^{(i u+\alpha) k}\left(e^{(r-d) \bar{T}+x}-e^{k}\right) d k d x \\
= & \frac{\exp \{[(r-d)(i u+\alpha+1)-r] \bar{T}\}}{(i u+\alpha)(i u+\alpha+1)} \\
& \times \int_{-\infty}^{\infty} e^{(i u+\alpha+1) x}\left(\rho_{X_{\bar{T}}}(x)-\rho_{B S_{\bar{T}}}(x)\right) d x \\
= & \frac{\exp \{[(r-d)(i u+\alpha+1)-r] \bar{T}\}}{(i u+\alpha)(i u+\alpha+1)} \\
& \times\left(\Phi_{X_{\bar{T}}}(u-i \alpha-i)-\Phi_{B S_{\bar{T}}}^{\sigma}(u-i \alpha-i)\right) .
\end{aligned}
$$

By the Fourier inversion of $\zeta_{T, t}(u)$, the equation (19) is obtained. Next we define the function $\hat{\zeta}(S)$ as

$$
\hat{\zeta}(S):=S \int_{-\infty}^{+\infty} e^{-(i u+\alpha) k} \zeta_{T, t}(u) d u .
$$


Let us differentiate $\hat{\zeta}_{T, t}(S)$ once and twice. Thus,

$$
\begin{aligned}
\frac{\partial \hat{\zeta}}{\partial S} & =\int_{-\infty}^{+\infty} e^{-(i u+\alpha) k} \zeta_{T, t}(u) d u+S \int_{-\infty}^{+\infty} \frac{\partial k}{\partial S} \frac{\partial}{\partial k} e^{-(i u+\alpha) k} \zeta_{T, t}(u) d u \\
& =\int_{-\infty}^{+\infty}(i u+\alpha+1) e^{-(i u+\alpha) k} \zeta_{T, t}(u) d u \\
\frac{\partial^{2} \hat{\zeta}}{\partial S^{2}} & =\int_{-\infty}^{+\infty} \frac{\partial k}{\partial S} \frac{\partial}{\partial k}(i u+\alpha+1) e^{-(i u+\alpha) k} \zeta_{T, t}(u) d u \\
& =\frac{1}{S} \int_{-\infty}^{+\infty}(i u+\alpha)(i u+\alpha+1) e^{-(i u+\alpha) k} \zeta_{T, t}(u) d u
\end{aligned}
$$

Therefore the equation (21) is obtained. 